\title{
Toward the actual model exchange using FMI in practical use cases in Japanese automotive industry
}

\author{
Yutaka Hirano $^{1}$, Junichi Ichihara ${ }^{2}$, Haruki Saito ${ }^{3}$, Yosuke Ogata ${ }^{4}$, Takayuki Sekisue ${ }^{5}$, Satoshi Koike ${ }^{6}$, \\ ${ }^{1}$ Toyota Motor Corporation, Japan \{yutaka_hirano@mail.toyota.co.jp\}, \\ ${ }^{2}$ AZAPA Co. LTD, Japan \{junichi-ichihara@ azapa.co.jp\}, \\ ${ }^{3}$ Nissan Motor Corporation, Japan \{haruki-s@mail.nissan.co.jp\}, \\ ${ }^{4}$ Siemens K.K., Japan \{yosuke.ogata@siemens.com\}, \\ ${ }^{5}$ ANSYS Japan K.K., Japan, \{takayuki.sekisue@ansys.com\}, \\ ${ }^{6}$ DENSO CORPORATION\{SATOSHI_L_KOIKE@denso.co.jp
}

\begin{abstract}
The working group in JSAE (Society of Automotive Engineers of Japan) proposed and published a guideline about a method using adapters to enable the connection of FMUs in acausal modeling tools in 2015. After that, more combination of exporting tools and importing tools of FMUs were tested for more realistic models. The success of the method was confirmed in the case of using FMI for Model Exchange (ME). Additionally the connection of FMUs generated from various modeling tools was tested using FMI for CoSimulation (CS) for a full-vehicle test model. Through the above-mentioned activities, we obtained much knowledge about utilizing FMI for practical model exchange in the industry. Finally the requests for future realization of FMI are described.
\end{abstract}

Keywords: FMI, Model Exchange, Co-Simulation, Benchmark Model, Numerical Stability

\section{Introduction}

Importance of utilizing simulation is increasing for the development of automotive systems. Both high functionality and high reliability are required while the development time is becoming shorter. For large-scale and multi-domain development of automotive systems, environment for connecting models developed in various organizations is increasing its importance (Sekisue et al, 2013). For this purpose, FMI (Functional Mockup Interface) is one of the most important technologies to realize the exchange of models developed by various tools. (Blochwitz et al, 2011) (FMI)

Upon above background, the Working Group about Research for FMI Utilization and Expansion in the Committee on Automotive Control and Model in JSAE has been continuing its efforts to 1) find problems about practical usage of FMI for exchanging models in actual development, 2) research about the measures to solve the problems, 3) make guidelines about utilizing FMI for exchanging models and 4) educate and encourage users about using FMI for exchanging models in Japan.
In the previous research a method to use special adaptor models to divide and make FMUs of the submodels and to connect FMUs in acausal modeling environment was proposed (Hirano et al, 2015). The method was validated by a benchmark model in the case of using ME. In this paper, many combinations of tools to create FMUs and connect FMUs were tested for the proposed method. There were some problems found in those tests not only when using ME but also when using CS. Another test model of a full vehicle model to connect FMUs of each sub-system of the vehicle using CS was tested in various combinations of the tools. Through those activities, we encounter the important points about connecting FMUs in the practical use cases such as handling of algebraic loops and stability of simulation for stiff systems. Finally future plans about our activities will be described.

\section{Method to use adaptor models}

In the previous research, a method to use adaptor models of electric domain and mechanical domain as shown in Figure 1 was introduced (Hirano et al, 2015).

\begin{tabular}{|c|c|c|}
\hline Electric & Rotational Mechanical & Translational Mechanical \\
\hline $\begin{array}{r}E_{\text {pin }}=v \\
I_{\text {pin }}=i\end{array}$ & $\begin{array}{l}\text { Torque signal output } \\
\varphi_{\text {flange }}=p h i \\
\omega_{\text {flange }}=w \\
a_{\text {flange }}=a \\
\tau_{\text {flange }}=\text { tau } \\
\varphi_{\text {flange }}, \omega_{\text {flange }}, a_{\text {flange }}, \tau_{\text {flange }}\end{array}$ & $\begin{array}{l}\text { Force signal output } \\
s_{\text {trfln }}=s \\
v_{\text {trfln }}=v \\
a_{\text {trfln }}=a \\
f_{\text {trfln }}=f \\
s_{\text {trfln }}, v_{\text {trfln }}, a_{\text {trfln }}, f_{\text {trfln }}\end{array}$ \\
\hline $\begin{array}{l}E_{\text {pin }}=v \\
I_{\text {pin }}=-i\end{array}$ & 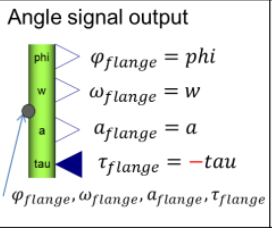 & \begin{tabular}{|l|l} 
Position signal output \\
$s_{\text {trfln }}=s$ \\
$v_{\text {trfln }}=v$ \\
& $a_{\text {trfln }}=a$ \\
$f_{\text {trfln }}=-f$
\end{tabular} \\
\hline
\end{tabular}

Figure 1. Adapter models to connect acausal physical port and causal signal ports 


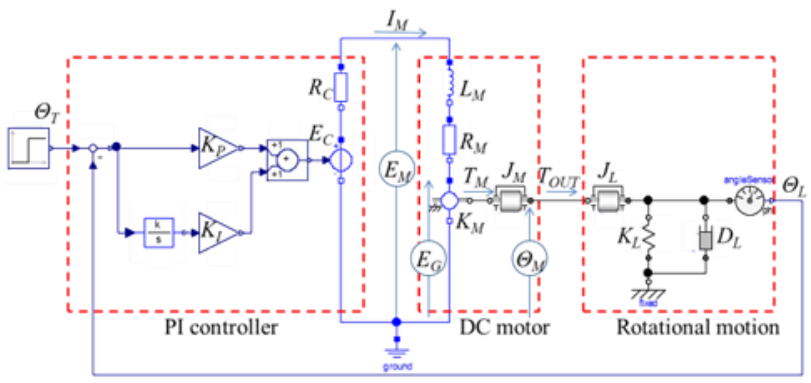

Figure 2. Benchmark model (Simple control system)

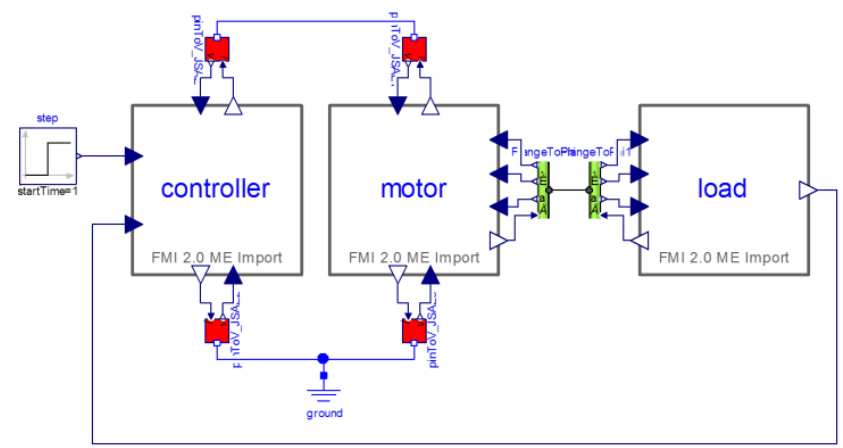

Figure 3. System model using 3 FMUs

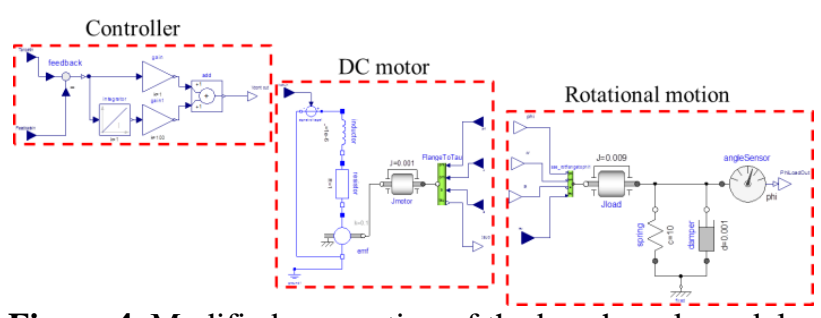

Figure 4. Modified connection of the benchmark model

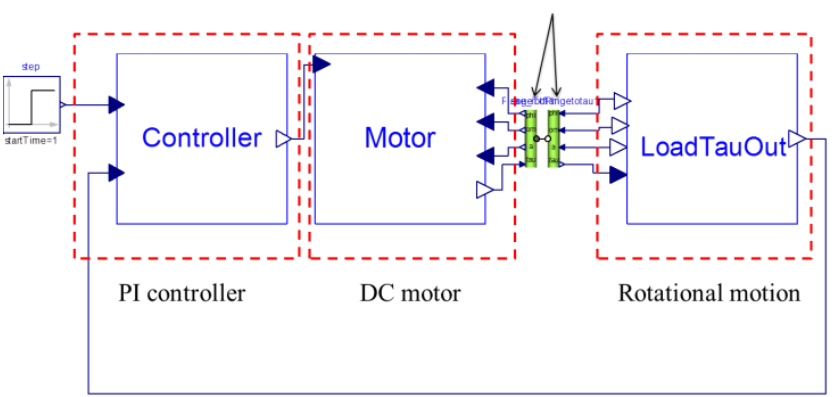

Figure 5. Modified benchmark model with 3 FMUs

This method was tested by a benchmark model shown by Figure 2. Three sub-systems of the benchmark model indicated by dotted square in Figure 2 were converted to FMUs by connecting the adaptor models for each inputs and outputs. Then three FMUs were connected by using corresponding adaptors of each inputs and outputs as shown in Figure 3. Simulation results of the original acausal model and the model using 3 FMUs were compared. It was confirmed that the results were almost identical. Though, in this case, only one Modelica tool was used to create and connect FMUs.

This time many combinations of the tools for creating FMUs and connecting FMUs were tested. First, it became clear that some combinations of tools failed to simulate the benchmark model when using the adaptors for electric domain. Thus, the benchmark model was a little modified about its connection. In this case, the electric connectors were not used but just controller command as the voltage input to the motor was used as shown in Figure 4. After generating FMUs from each subsystem of Figure $\mathbf{4}$ by various tools, each FMUs were connected in the system model as shown in Figure 5. Five tools were used to create FMUs and four tools were used to connect FMUs. Finally the result of whether the simulation worked became as shown in Table 1. Here, the meanings of the symbols in each column are as follows.

- for 'adaptor':

$\Delta$ - Contains only adaptors for some physical domain

$x$ - Could not create adaptor models

- for 'Run':

\section{$\Delta$ - Do not use adaptors and connect directly}

The number in the lower part of each cell means the ratio of calculation time of each model with FMUs compared with that of the original acausal model.

Almost all tools could create and run FMUs using the adaptor models. Only Tool D could not create adaptor models. Also Tool D could run FMUs created by other tools by connecting causal signals directly but not using the adaptors. Also it became clear that the calculation time became very long in some combinations of the tools. The reasons of this phenomenon were assumed as 1) that the compatibility of the master algorithm was bad for the embedded FMUs (ex. the algorithm for solving the algebraic loops and /or solving the stiff systems), 2) difficulty of determining the initial values when the re-initialization occurred, 3) improper setting of the simulation parameters of the solvers, and so on. But the details are not clear yet because these investigations need deep insight of the algorithms of each tool but it is not easy for the users' side.

Table 1 Results of connecting FMUs and run

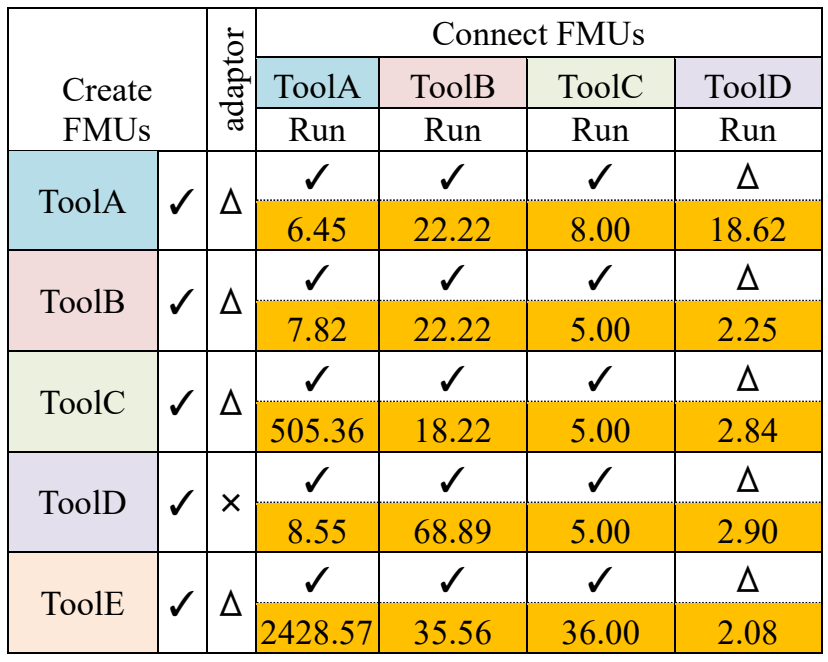


Above results were obtained in the case of using ME. We also tried to test same cases by using CS. But for $\mathrm{CS}$, it became clear that the adaptor model of the rotational mechanics domain using all of rotational angle, velocity and acceleration resulted in the simulation error because of over constrained condition. Thus it was suggested that we should modify the adaptor model to use just one state variable of rotational motion as the across variable. This method will be investigated in near future.

Additionally there was a remark from Modelica Association that adaptor models in many physical domains have been added to the master branch of the Modelica Standard Library (MSL) (https://github.com/modelica/Modelica) and will become available in the next release of the MSL.

\section{Connection of full vehicle model using CS}

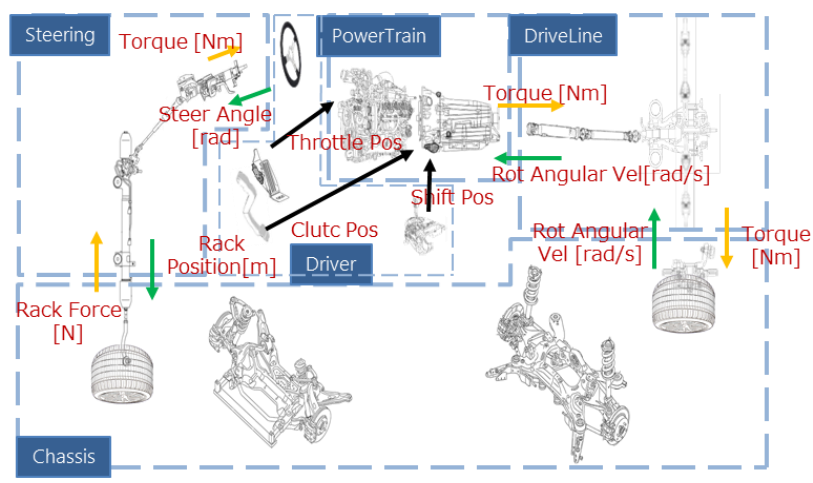

Figure 6. Full vehicle benchmark model

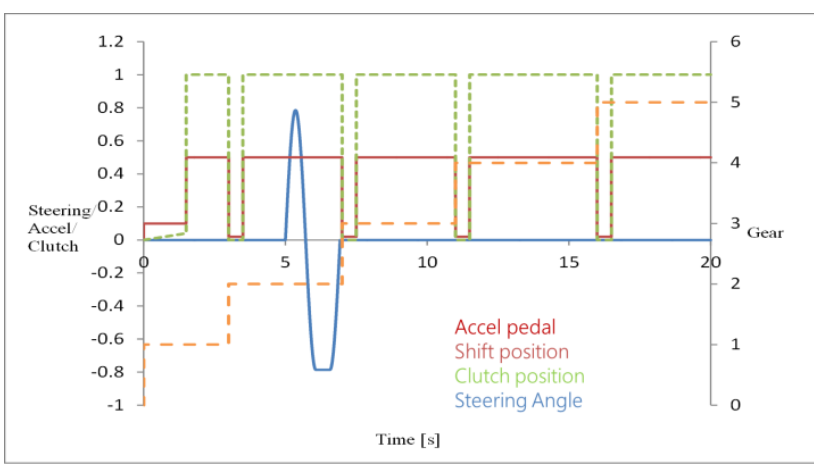

Figure 7. Driver's maneuver inputs to the full vehicle model

Table 2 Characteristics of each sub-system of the full vehicle model

\begin{tabular}{|l|l|l|}
\hline Sub-system & Number of state variables & Dynamics \\
\hline Driver & 0 & - \\
\hline Power train & 63 & $2.3[\mathrm{kHz}]$ \\
\hline Drive line & 6 & $3.2[\mathrm{kHz}]$ \\
\hline Chassiss & 40 & $10[\mathrm{~Hz}]$ \\
\hline Steering & 12 & $240[\mathrm{~Hz}]$ \\
\hline
\end{tabular}

For the research of connecting sub-systems of the full vehicle model by $\mathrm{CS}$, the benchmark model shown in Figure 6 was used. In this case, signals between FMUs were connected directly without using the adopter models mentioned above. Sub-systems consist of the steering system, the powertrain system, the driveline system, the chassis system and the driver model. Physical variables transferred between each sub-system and their directions are shown in Figure 6. This selection of physical variables and their directions were decided by the guideline about the model interface of full vehicle model written by a Working Group of METI (Ministry of Economics, Trade and Industries) of Japanese Government. (METI, 2017)

Driver's open loop maneuver inputs to the full vehicle model were decided as shown in Figure 7. Table 2 shows the number of the state variables and maximum frequency of the dynamics of each subsystems. Simulation tests were done by following sequences.

1. Simulate the full vehicle model of original acausal model in one host tool (Tool A).

2. Replace each sub-system model to FMUs generated by the host tool (Tool A) and simulate the full vehicle model with replaced FMUs in the host tool (Tool A).

3. Replace the sub-system models to FMUs generated by different tools of the host tool. Each tool to generate the FMUs of sub-systems are shown in Table 2. Then simulate the changed model in the host tool (Tool A).

4. Change the host tool of the full vehicle model to Tool B, C and D using FMUs from the different tools mentioned in the above sequence 3 .

Here, tested host tools (master of CS) are as follows. Tool A: Amesim, Tool B: Simulink + Modelon FMI Toolbox, Tool C: Dymola, Tool D: SimulationX. (Please note that these tools are different from that of the chapter 1.)

Table 3 shows the various test conditions for the above test sequence 1 and 2. Both of the variable step solver (LSODA) and the fixed step solver (RK4) were compared. Figure 8 shows one example of the results. There were some oscillation caused by the stepwise change of the driver's maneuver inputs. But the results of all the cases were almost identical regardless of whether FMUs were used or not. Also the results were not changed so much by the changes of the simulation algorithm and its parameter if the integration step size and the communication step size (described as 'interval' in the table) of FMUs were small enough. Also for the above test sequence 3 and 4, many cases of changing integration algorithm and communication step size were tested as shown in Table 4 . The results of all the cases in Table 4 were almost identical. But there were a slight change in the oscillation of the results as 
shown in Figure 9 when the communication step size of FMUs was changed. It was confirmed that larger oscillation of the simulation result appeared for the larger communication step size. As shown in Table 4, there were trade-off between the calculation time of the host tool and the preciseness and stability (oscillation) of the simulation results according to the selection of the integration algorithm, its calculation interval and the communication step size of FMUs by CS. Figure $\mathbf{1 0}$ shows one simulation result of the sequence 4 . The results of the simulation were almost identical when different CS master tool were used.

Table 3. Simulation test conditions of the full vehicle benchmark model (Sequence 1 and 2)

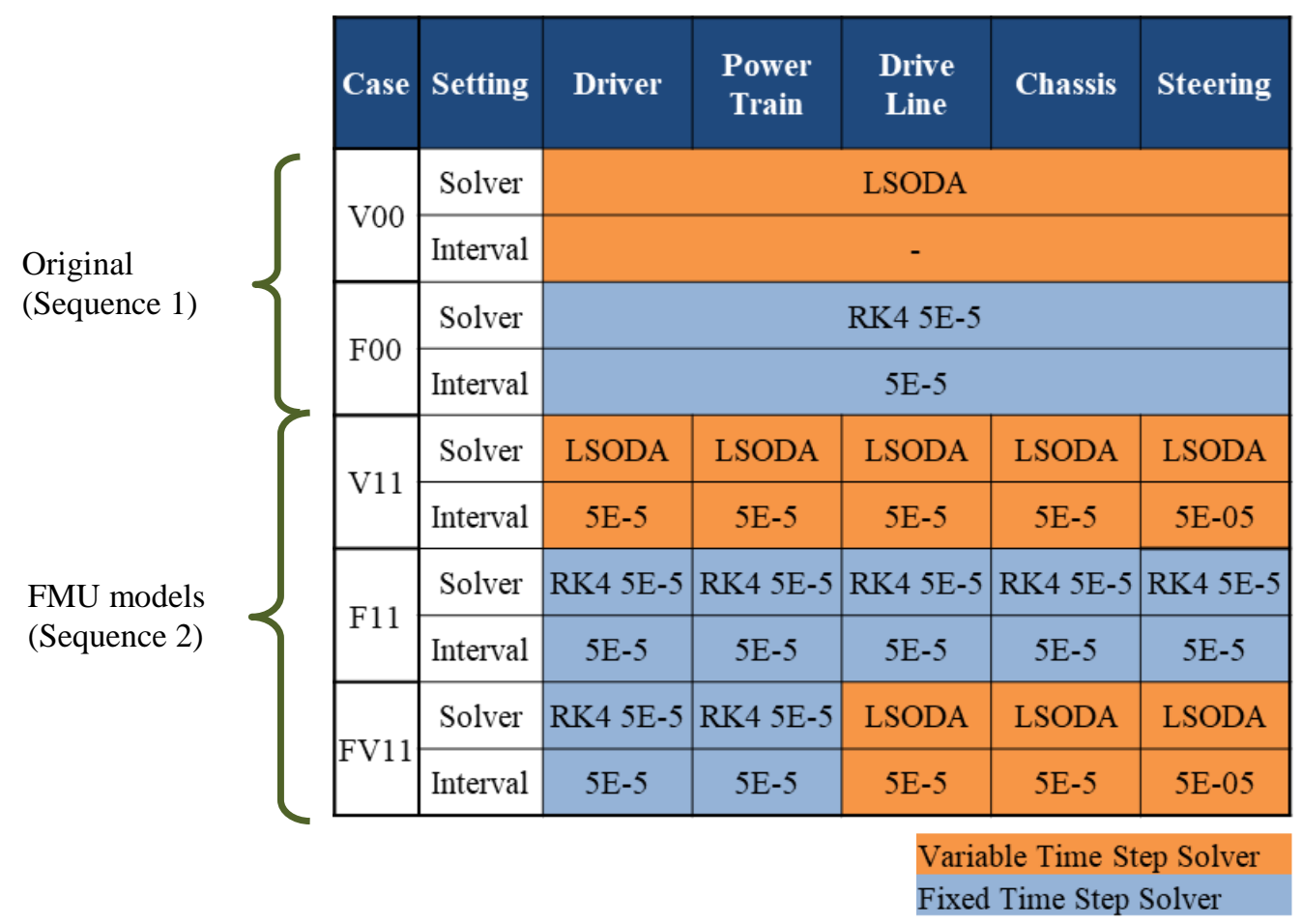
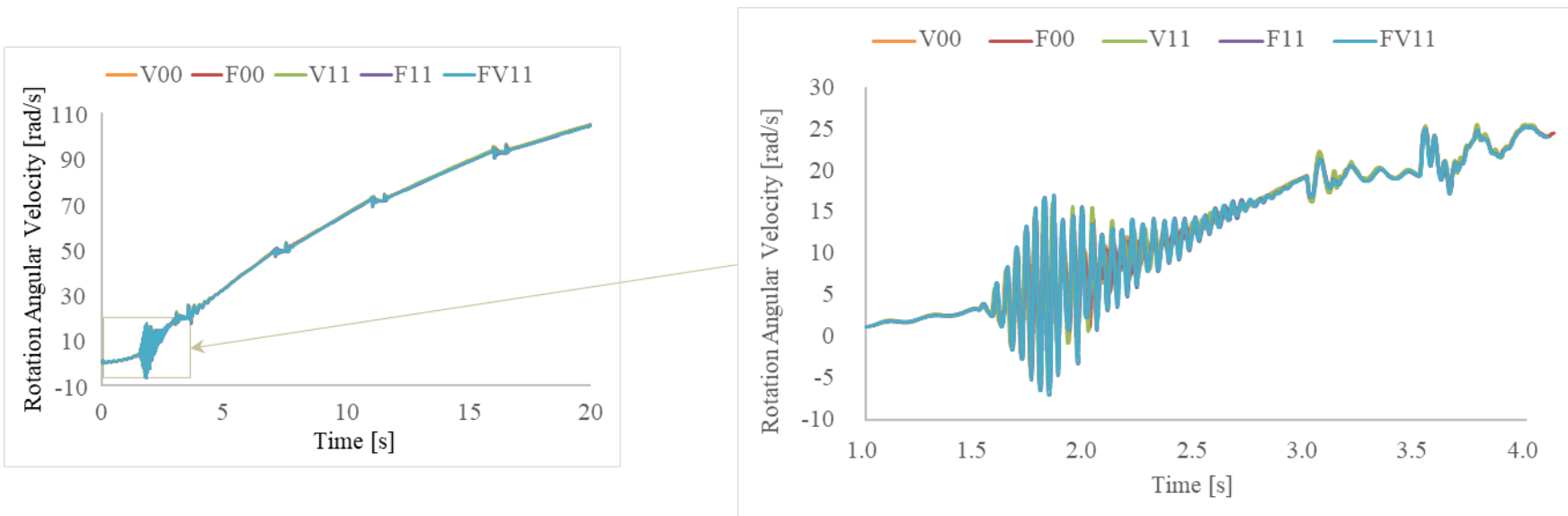

Driveshaft Rotation Angular Velocity (Chassis)

Figure 8. Simulation results of the full vehicle model (Sequence 1 and 2) 
Table 4. Simulation test conditions of the full vehicle benchmark model (Sequence 3 and 4)

\begin{tabular}{|c|c|c|c|c|c|c|c|}
\hline \multirow{3}{*}{ Case } & \multirow{3}{*}{ Setting } & \multicolumn{5}{|c|}{ Slave Tool FMU } & \multirow{2}{*}{\begin{tabular}{|l}
$\begin{array}{l}\text { Master Tool } \\
\text { CPU time* }\end{array}$ \\
$\begin{array}{l}\text { Tool A } \\
\text { (Amesim) }\end{array}$
\end{tabular}} \\
\hline & & $\begin{array}{l}\text { Tool A } \\
\text { (Amesim) }\end{array}$ & $\begin{array}{l}\text { Tool A } \\
\text { (Amesim) }\end{array}$ & $\begin{array}{l}\text { Tool B } \\
\text { (Simulink + } \\
\text { Modelon Tool) }\end{array}$ & $\begin{array}{l}\text { Tool C } \\
\text { (Dymola) }\end{array}$ & $\begin{array}{l}\text { Tool D } \\
\text { (SimlationX) }\end{array}$ & \\
\hline & & Driver & Power Train & Drive Line & Chassis & Steering & Rk4, 5e-5 \\
\hline \multirow{2}{*}{1} & Solver & RK4 5E-5 & RK4 5E-5 & RK4 5E-5 & RK4 1E-3 & CVODE & \multirow{2}{*}{6.2} \\
\hline & Interval & $5 \mathrm{E}-5$ & $5 \mathrm{E}-5$ & $5 \mathrm{E}-5$ & $1 \mathrm{E}-3$ & $1 \mathrm{E}-3$ & \\
\hline \multirow{2}{*}{$2-1$} & Solver & RK4 5E-5 & RK4 5E-5 & RK4 5E-5 & RK4 5E-5 & CVODE & \multirow{2}{*}{42.5} \\
\hline & Interval & $5 \mathrm{E}-5$ & $5 \mathrm{E}-5$ & $5 \mathrm{E}-5$ & $1 \mathrm{E}-3$ & $1 \mathrm{E}-3$ & \\
\hline \multirow{2}{*}{$2-2$} & Solver & RK4 5E-5 & RK4 5E-5 & RK4 5E-5 & RK4 5E-5 & CVODE & \multirow{2}{*}{44.6} \\
\hline & Interval & $5 \mathrm{E}-5$ & $5 \mathrm{E}-5$ & $5 \mathrm{E}-5$ & $5 \mathrm{E}-4$ & $5 \mathrm{E}-4$ & \\
\hline \multirow{2}{*}{$2-3$} & Solver & RK4 5E-5 & RK4 5E-5 & RK4 5E-5 & RK4 5E-5 & CVODE & \multirow{2}{*}{86.4} \\
\hline & Interval & $5 \mathrm{E}-5$ & $5 \mathrm{E}-5$ & $5 \mathrm{E}-5$ & $5 \mathrm{E}-5$ & $5 \mathrm{E}-5$ & \\
\hline \multirow{2}{*}{$3-1$} & Solver & RK4 5E-5 & RK4 5E-5 & RK4 5E-5 & DASSL & CVODE & \multirow{2}{*}{129.7} \\
\hline & Interval & $5 \mathrm{E}-5$ & $5 \mathrm{E}-5$ & $5 \mathrm{E}-5$ & $1 \mathrm{E}-3$ & $1 \mathrm{E}-3$ & \\
\hline \multirow{2}{*}{$3-2$} & Solver & RK4 5E-5 & RK4 5E-5 & RK4 5E-5 & DASSL & CVODE & \multirow{2}{*}{264} \\
\hline & Interval & $5 \mathrm{E}-5$ & $5 \mathrm{E}-5$ & $5 \mathrm{E}-5$ & $5 \mathrm{E}-4$ & $5 \mathrm{E}-4$ & \\
\hline \multirow{2}{*}{$3-3$} & Solver & RK4 5E-5 & RK4 5E-5 & RK4 5E-5 & DASSL & CVODE & \multirow{2}{*}{2716} \\
\hline & Interval & $5 \mathrm{E}-5$ & $5 \mathrm{E}-5$ & $5 \mathrm{E}-5$ & $5 \mathrm{E}-5$ & $5 \mathrm{E}-5$ & \\
\hline
\end{tabular}

* CPU time shows the ratio of calculation time standardized by that in the case of acausal original model

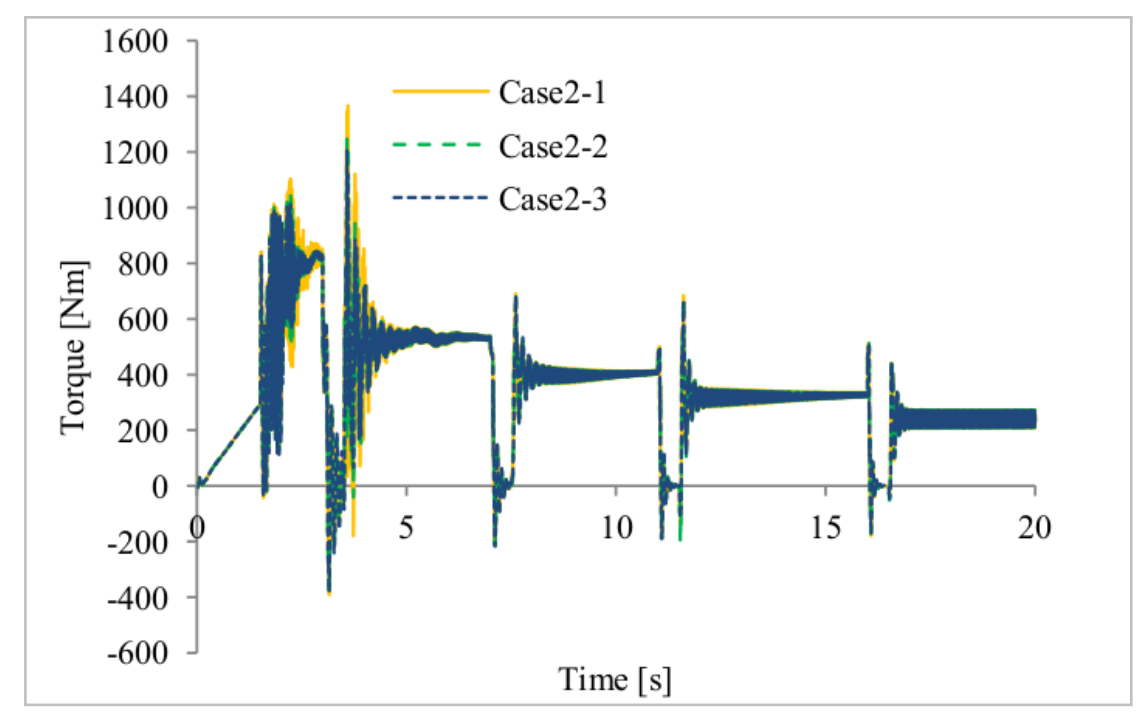

Rear Left Driveshaft Torque (DriveLine)

Figure 9. Simulation results of the full vehicle model for the different communication step size 


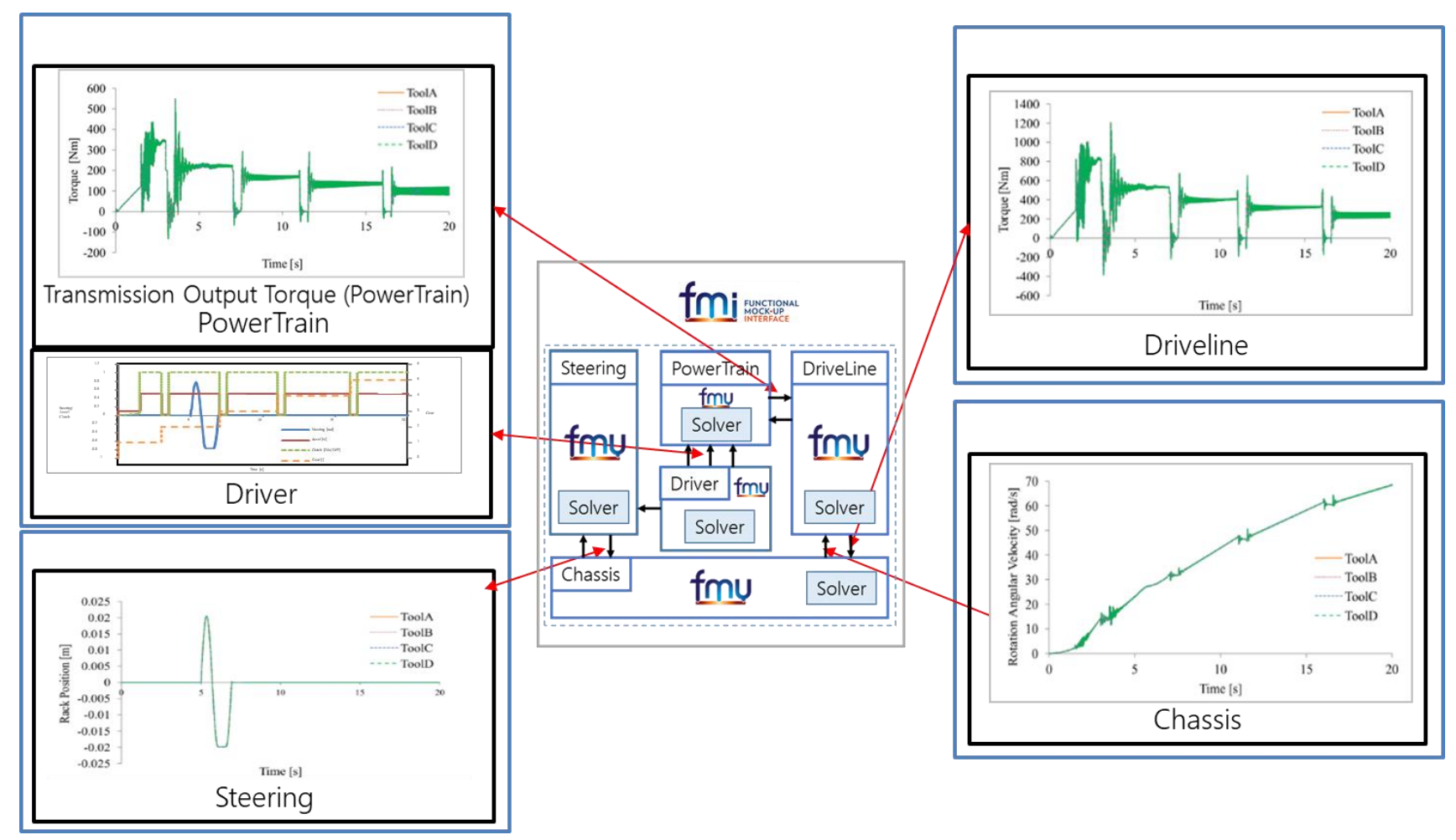

Figure 10. Simulation result of the full vehicle model (Sequence 4, Case 3-3)

\section{Important points about connecting FMUs}

Through the above researches, we obtained some knowledge and important points about connecting models by FMI. There are three major phases when we try to connect and exchange models by FMI.

\subsection{Creation and export / import of FMU}

Sometimes there still occurred errors by inconsistency between tools about exchanging information about the models.

1) Some tools could not read the XML information when there were @ character in the name of input / output / state variables.

2) There were inconsistency about type and unit of variables between some tools.

It is desirable that those specifications will be unified between every tool and also checked by FMU compliance checker as soon as possible.

\subsection{Connection of FMUs in the host tool}

Even when the import and connection of FMUs was successful, there were problems of inconsistency about the direction and sign of the signals exchanged between FMUs and the host tool. This is because of that FMI is based on the causal signal interconnection. It is necessary that those specifications should be identical and agreed with the both parties of exporting FMUs and connecting FMUs.

For the full vehicle model, the Working Group of METI about unifying the definition of signals exchanged between automotive systems has recently announced the standard guideline of the model interconnection for automotive industries in Japan. The benchmark models of our research also keep this guideline. (Some members of both Working Groups of METI and JSAE are same.)

\subsection{Stability and preciseness of the simulation}

When running the simulation, there are many points we should be careful. The points differ between the case of $\mathrm{ME}$ and CS.

\subsubsection{In the case of using ME}

There are mainly three problems when using ME.

1) Generation of algebraic loop

In the case of ME, there is no time delay between the inputs and outputs of FMUs. Thus there occurs a possibility that the 'real' algebraic loop is generated when connecting different FMUs as shown in Figure 11. On the other hand, in the case of CS, the possibility of the generation of the algebraic loop is low because there always is time delay of communication step size as shown in Figure 11. Here, the 'artificial algebraic loop' means that if there is no information about the causality of inputs and outputs of FMUs provided as the additional 
information by XML file, the solver of the master tool tries to solve the loop by iterative loop solver algorithm. This can be happened in the both cases of CS and ME.

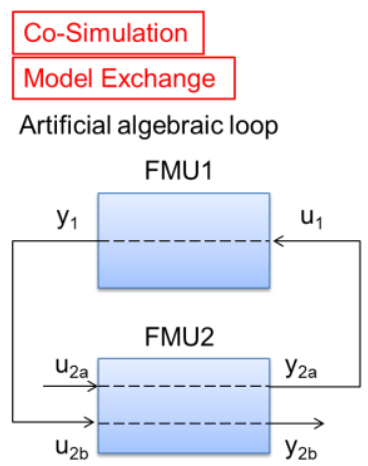

Model Exchange

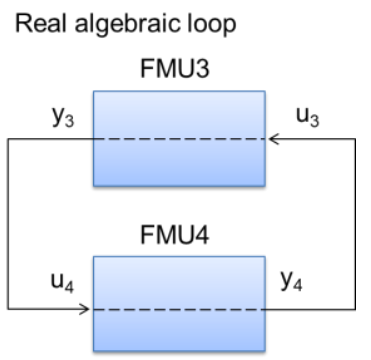

Figure 11. Two types of the algebraic loop

When the real algebraic loop occurs in the case of using $\mathrm{ME}$, it is necessary to solve by some algebraic loop solver using an iteration method. Whether it would be successful to solve the algebraic loop and also the efficiency and the correctness of the simulation heavily depends on the performance of the algebraic loop solver of each host tool. Also some users try to avoid the algebraic loop by inserting time delay unit into the loop. Though this method is dangerous. If the absolute value of the loop gain exceeds one, then the calculation will diverse.

2) Selection of the solver

Because only the solver of the host tool which import FMUs can be used when using ME, the selection of the solver for the numerical integration is very important. If the time constants of each FMU differs widely, then the total system connecting FMUs becomes stiff system. In such a case, it is necessary to use an integration algorithm which can cope with the stiff system. Also the parameter of the solver such as tolerance and/or step size should be selected carefully. When using fixed step solvers, the step size should be selected so that all the eigenvalue of the system should be within the stable region of z-plain as shown in Figure 12. Here, $\lambda$ is the minimum eigenvalue and $\Delta \mathrm{t}$ is the calculation step size. However, in the current situation, there is no guideline or suggestion about the selection of the proper solver and its parameters. This results in the difficulty of using FMI for ME as the method of connecting and exchanging the FMUs between different organizations. One remedy would be to make the output of the information about the time constants such as Jacobian as mandatory and let the every tool to use this information to recommend the best selection of the solver and its parameter to the users.

3) Initialization and event handling

Inconsistency of initial values of the state variables between FMUs is also a problem. Currently it is not mandatory to specify the initial values of the state variables. It is highly desired that this specification would be unified and become mandatory for all the tools. Also the ability of event detection and reinitialization of the state variables is not same between tools. Sometimes this results in the difference of the simulation results.

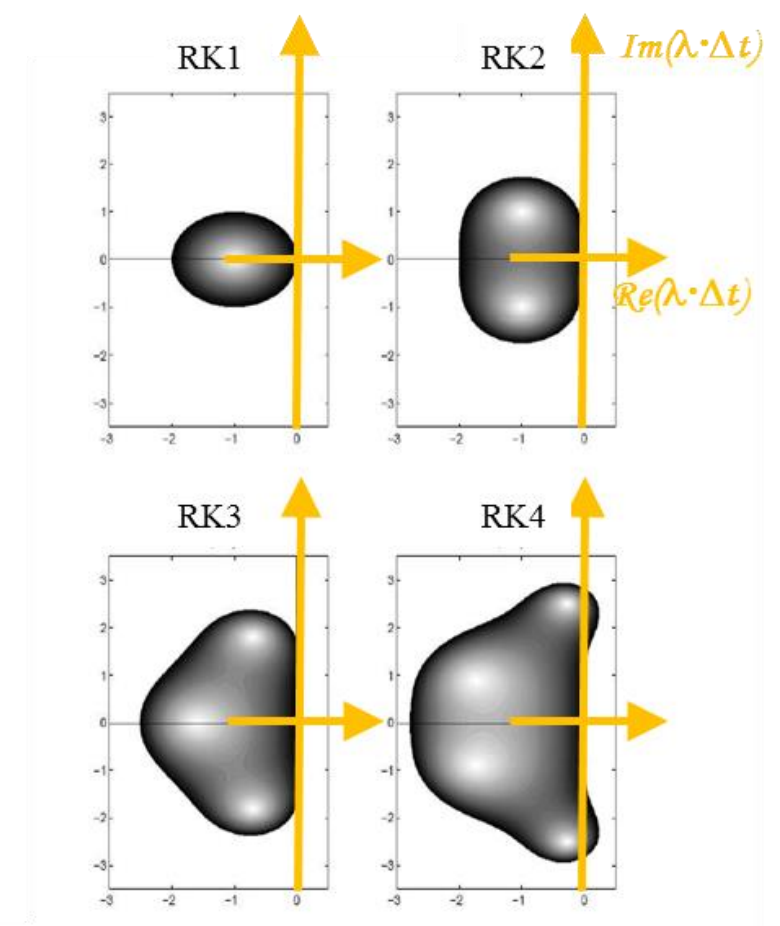

Stable zone of Runge Kutta solver (Order 1 to 4)

Figure 12. Stable zone of Runge Kutta solver

\subsubsection{In the case of using CS}

As shown in the previous sub-section, there is no possibility that the real algebraic loop would be generated when using CS. Also because each FMU has the proper solver from the master tool (if it was tested well), the problem of the selection of the solver is not so difficult as in the case of using ME. It is supposed that the benchmark test using full vehicle model by CS mentioned in chapter 3 worked well because of these reasons.

However, there always occurs the problem of time delay of communication step size (CSS) for CS. Because the dead time of CSS acts same as the sample and hold unit of a discrete system, the simulation tend to become unstable if the CSS is too big. Concerning the selection of CSS, there is a trade-off between calculation time and the preciseness of the simulation as mentioned in chapter 3 . To consider the proper value of the CSS, the information about the eigenvalues of each FMU is also important as same as in the case of ME. The measure to solve this problem would be same as mentioned above. 


\section{Future plans}

To figure out what will be necessary for our future plan, we gathered answers of questionnaires from the members of JSAE and tool users. Figure 13 shows the distribution of belonging industries of the respondents. Figure 14 shows the current status of on-the-job usage of FMI of them. About 22\% of the respondents were utilizing or trying FMI. On the other hand, $31 \%$ of them didn't know FMI yet.

\section{Industries ( $\mathrm{N}=172)$}

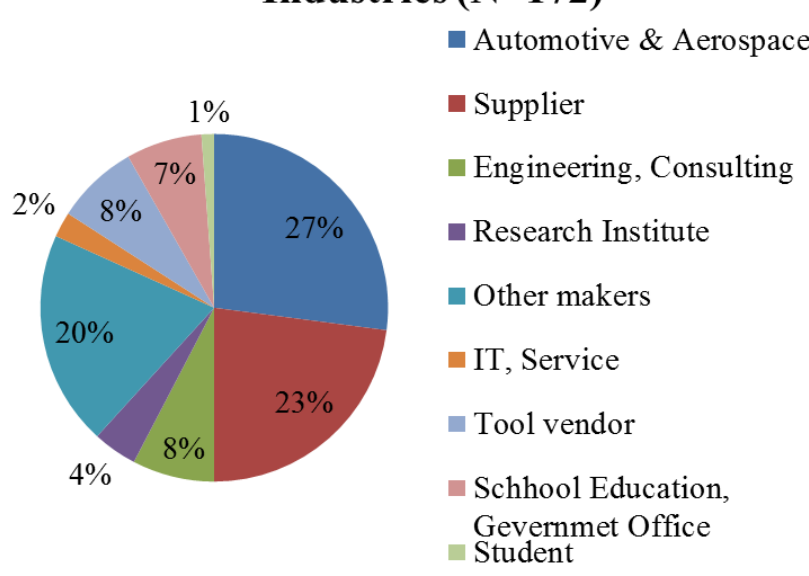

Figure 13. Distribution of industries of respondents

\section{On-the-job Usage of FMI $(\mathrm{N}=172)$}

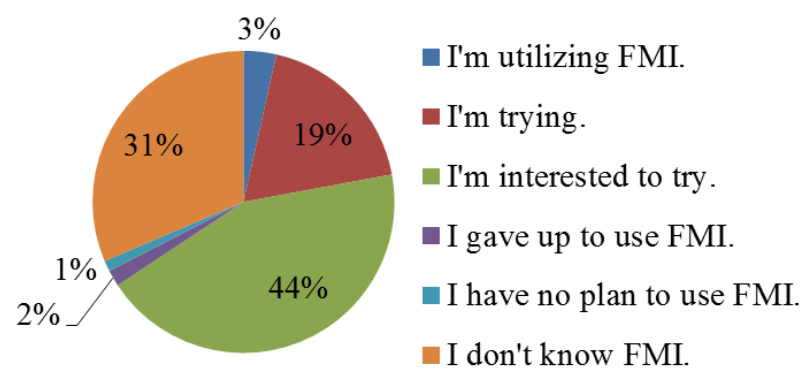

Figure 14. On-the-job usage of FMI

For the actual users of FMI, the purpose of using FMI was as shown in Figure 15. (Multiple number of the answers were possible.) It became clear that about $40 \%$ was for model exchange between organizations by either of ME or CS. Also utilization for HIL / Real time simulation was higher than expected. Figure 16 shows problems and requests about FMI from the respondents. The similar problems with our research such as the problems about numerical solvability and stability, inconsistency of parameters and signal specification were reported. Also the requests about speed-up of calculation and large scale connection of FMUs including hierarchical connection were clarified. Considering these problems and requests, we are extending the activity of our Working Group to 1) extension of physical region to evaluate (to include thermal system and fluid system), 2) Coping with hierarchical connection and speed-up of simulation, 3) Updating JSAE guideline, 4) Collaborating more with the related groups such as METI, Modelica Association FMI Project, etc. and 5) Organizing seminars and an Organized Session of JSAE Conference to educate and expand knowledge of FMI users in Japan.

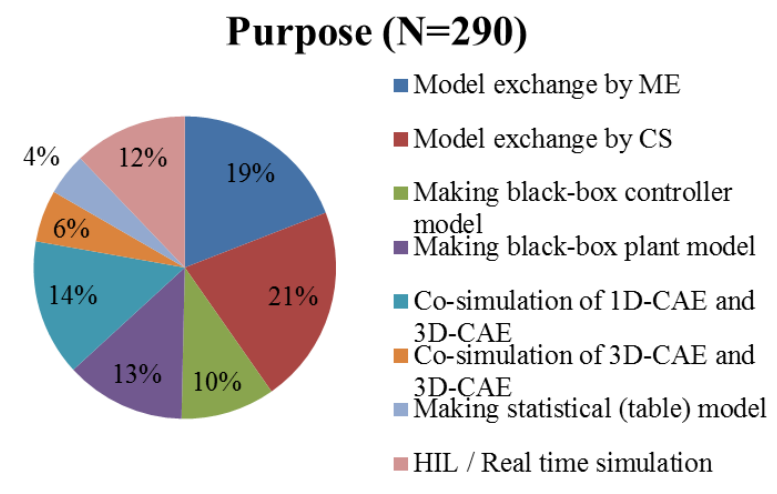

Figure 15. Purpose of utilizing FMI

\section{Problems and Requests $(\mathrm{N}=167)$}

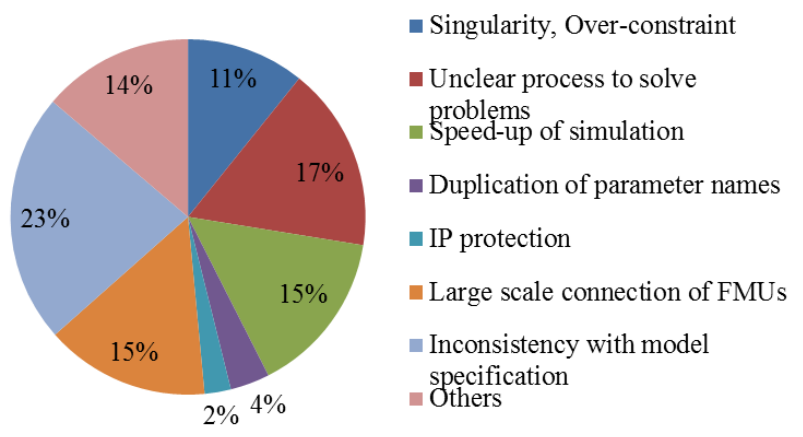

Figure 16. Problems and requests about FMI

\section{Conclusion}

Many tests using benchmark models for both of the cases of using ME and using CS were done to confirm the properness of using FMI for connecting and exchanging models in automotive industry in Japan. Though there still are many remained problems about using FMI for practical model exchange, most of the tests were successful. Also the reasons of the problems were estimated and countermeasures for those problems were proposed. It is highly desirable that the proposal from us will be realized in the future specification of FMI and also in the implementation of the related tools. 


\section{References}

T. Blochwitz, M. Otter, M. Arnold, C. Bausch, C. Clauß, H. Elmqvist, A. Junghanns, J. Mauss, M. Monteiro, T. Neidhold1, D. Neumerkel, H. Olsson, J.-V. Peetz, S. Wolf, The Functional Mockup Interface for Tool independent Exchange of Simulation Models, Proc. of the $8^{\text {th }}$ International Modelica Conference, 2011

FMI. The functional mockup interface. http://www.functional-mockup-interface.org/.

Y. Hirano, S. Shimada, Y. Teraoka, O. Seya, Y. Ohsumi, S. Murakami, T. Hirono, T. Sekisue, Initiatives for acausal model connection using FMI in JSAE, Proc. of the $11^{\text {th }}$ International Modelica Conference, 795-801, 2015. Doi:10.3384/ecp15118795

JSAE Committee on Research of Model Development and Circulation Methods Based on Global Standardized Description, Guideline of Model Connection using FMI in Acausal Modeling Tools, 2014 (in Japanese. Available online as http://www.jsae.or.jp/tops/topics/1241/12411A.pdf).

METI (Ministry of Economics, Trade and Industry) of Japan, The Study Group for Ideal Approaches to Model Utilization in the Automobile Industry Releases a Compilation of Discussion Results, 2017 (Available online as

http://www.meti.go.jp/english/press/2017/0331_004.html, Guidelines are available online but only in Japanese as http://www.meti.go.jp/press/2016/03/20170331010/20170 331010-1.pdf and http://www.meti.go.jp/press/2016/03/20170331010/20170 331010-3.pdf)

T. Sekisue, K. Tsuji, M. Ogawa, T. Fukada, K. Tanimoto, S. Hikida, M. Ueda, T. Kato, Alternator model for full vehicle simulation, Proc. JSAE annual conference 2013 Spring, No.407-20135490, 2013 (in Japanese). 